\title{
ATENCION DEL PREMATURO POR EL OBSTETRA
}

\author{
Doctor Ernesto Plata Rueda \\ Ex-Jefe de la Sala del Instituto Materno-infantil C. V. A. \\ Conferencia dictada en la Universidad de América en el curso de obstetricia \\ para post-graduados. Abril de 1956. \\ 10 Definiciones. \\ 29 El cbstetra ante el problema de la mortalidad infantil. \\ 3 o Causas y prevención de la prematuridad. \\ 4 : El parto del prematuro. \\ 50 Cuidados inmediatos del prematuro. \\ 6 ? Las primeras 24 horas del prematuro. \\ 7 ? Generalidades sobre atención de prematuros.
}

10 Definiciones.-Por convenio universal se ha establecido que niño prematuro es aquel cuyo peso al nacimiento es inferior a 2.500 gramos, con prescindencia de la edad de la gestación (no siempre iácil de apreciar), talla y otras medidas (de uso poco práctico) y sin tener en cuenta tampoco su esatdo de salud, de único o múlti. ple ni su grado de vitalidad (de apreciación puramente subjetiva). Es con esta base como la estadística ha podido contar con una base muy firme para apreciar cifras de morbilidad y mortalidad. En esta forma el término débil congénito casi ha desaparecido de las clasificaciones modernas. Personalmente acostumbramos reservarlo para aquellos niños no clasificados como prematuros por tener 2.500 gramos o más, pero que exhiben en su vitalidad, aspecto y comportamiento ante el medio exterior, semejanzas con el verdadero prematuro. Illpo (1) sugirió en 1947 hacer una subdivisión y llamar inmaduros a los niños de peso inferior a 1.250 gramos 
cuya atención y pronóstico se apartan considerablemente de ios ciemás prematuros. Esta concepción ha sido aceptada parcialmente y en el Standard Nomenclature of Diseases and Operations, publicado por la Asociación Médica Americana (2), se establece el término inmaduro para los niños que pesan menos de 1.000 gramos. De acuerdo con la gestación los prematuros están entre las 28 y las 37 semanas y los inmaduros entre las 22 y 28 semanas.

$2^{\circ}$ El obstetra ante el problema de la mortalidad infantil.- La mortalidad infantil ha sufrido un descenso vertical a partir de 1912. Pero si se subdivide por grupos de edades, la única que prácticamente no se ha modificado es la del primer día (de 11 por mil a 9 por mil). Dado que la mortaidad en otras edades si ha descendido, resulta que la del primer dia, relativamente ha aumentado; de cada 100 muertos en 1912, 10 ccurr an en el primer dia; actualmente 30 ocurren en el primer día. Mueren más niños en los primeros 3 días que en los 362 restantes. Más de la mitad de la mortalidad de ios tres primeros dias ocurren en las prime. ras 24 horas; una tercera parte de esta mortalidad ocurre en las primeras 8 horas y más de la mitad de ésta en la primera hora. Más de la mitad de las muertes de los tres primeros días corresponden a niños menores de 1.500 gramos. El $70 \%$ de las muertes de los tres primeros días se debe a dos entidades: traumatismo obstétrico y ventilación pulmonar anormal, esta úitima que afecta especialmente a los prematuros. Estas cifras (resultado de 10.000 autopsias en Chicago) (3) son solo para poner de manifiesto cómo dada la elevada proporcićn que corresponde a los recién iacidos y especialmente a los prematuros en la mortalidad infantil generai y especiaimente por causas relacionadas con el embarazo y el parto, es evidente que el obstetra debe desempenar por fuerza un papel de primerisima importancia en toda campaña para disminuír la mortalidad infantil.

Ocurre sin embargo que hay en nuestro pais, un rigorismo exagerado, y un concepto equivocado sobre la especialización aleja cada día más al obstetra del niño, con las consecuencias que son de suponer. El obstetra puede y tal vez debe en ciertos medios delegar desde un principio la atención del niño en el pediatra; pero como ello no siempre es posible, debe tener la suficiente preparación pediátrica que le permite atender adecuadamente al niño y especialmente al prematuro, por lo menos en el "día de la 
muerte" como ha dado en llamarse a las primeras 24 horas del recién nacido. Es esta ia finalidad de nuestra charla sobre prematuros en este Curso de Obstetricia.

30 Causas y prevención de la prematuridad-Cualquiera se sorprende con el dato ya anotado de que la mortalidad de los tres primeros das casi no se haya modificado desde 1912 para acá, a pesar de los adelantos de la técnica médica. Entre las muchas causas que se han invocado se destaca la imposibilidad de luchar contra muchas de las deformidades congénitas y contra las le. siones de anoxia y traumatismo producidas por el parto, así como tambín el aumento de la incidencia de prematuridad como consecuencia de las exigencias de la vida moderna para la mujer. El aumento de los prematuros ha recargado los servicios a ellos desunados en tal forma que instituciones tan bien organizadas como la Casa dei Niño en Montevideo ha visto aumentar sus índices de mortalidad en los últimos años (4). Enfrentados como estamos a una mortalidad copada en un $70 \%$ por entidades poco modificables con tratamiento curative (ventilación pulmonar anormal y traumatismo intracraneano) y establecido que la mayoria de sus víctimas son prematuros, es evidente que el camino más útil a seguir es la prevención de la prematuridad io cual es de competencia del obstetra.

Toda campaña de prevención debe averiguar las calisas 0 factores de prematuridad que pueden clasificarse en medica: y económico-sociales. En orden aproximado de frecuencia e importancia las causas médicas son: toxemias, afecciones hemorragíparas del último trimestre, enfermedades médicas y quirúrgicas intercurrentes con el embarazo, embarazo múltiple, anomalías fetales, prematuridad habitual, edad de los padres, multipa. ridad, desequilibrios hormonales.

No nos compete como pediatras analizar estas causas a cuya corrección dedican diariamente sus esfuerzos los obstetras estudiosos. Solameste queremos destacar que la única manera de hacer que los resultados de las laboriosas investigaciones llevadas a cabo en los diversos terrenos enumerados, redunden en beneficios evidentes para la madre y el niño, en el control prenatal científico y juiciosamente orientado hacia la obtención de determinadas metas, y no la rutina de unas medidas y una prescripción para el vómito a que con tanta frecuencia se reduce el "control prenatal" en muchas instituciones. El control prenatal, que no debe ser inferior a 4 consultas, debe atender los siguientes puntos: 
el estado nutritivo de la madre, el descubrimiento precoz de enfermedades intercurrentes y su adecuado tratamiento (especial énfasis sobre cardiopatías, diabetes, nefropatias, tuberculosis, sífilis), el análisis del embarazo como normal o anormal y sus po. sibilidades para el futuro, la corrección de desequilibrios hor. monales, la información, educación y preparación anímica de la madre para el parto.

Los factores económico-sociales de la prematuridad constituyen otro gran capítulo y solo vamos a enumerarlos, pues es labor del médico solo ponerios de presente ante el Estado que es quien debe corregirlos. Casi todos en último término son de orden económico; alimentación carencial, trabajo de la embarazacia, vivienda y abrigo inadecuados, ilegitimidad, etc.

Vamos a extendernos más bien sobre el común denominador de todos estos factores que es la alimentación. El viejo concepto de que el feto se nutre satisfactoriamente a expensas de la madre cualquiera que sea la nutrición de ésta ha sido revaluado por las investigaciones modernas (5) que han establecido de manera concluyente como sobre todo en el último trimestre el niño compite inclusive en desventaja con la madre cuando ella no se alimenta adecuadamente. Una observación que se ha corroborado ya en todos los países y desde luego entre nosotros, es que las instituciones de maternidad se clasifican en el tipo socio_económico de las gentes que a ellas concurren por el peso de los niños y el índice de prematurez. Nuestras estadísticas en el Instituto Materno Infantil de Bogotá dan un índice de prematurez de $18 \%$. mientras otros establecimientos concurridos por madres de un nivel socio-económico superior dan cifras entre 6 y $10 \%$. Numerosas causas concurren a esta diferencia, casi todas en relación con las dificultades económicas. Sin embargo, una experiencia de post-guerra en Inglaterra (6) ha venido a poner de presente que de todos estos factores, la alimentación ejerce una influencia evidentemente superior. En efecto, durante la guerra (1939-1945), bajaron los indices de prematuridad y mortalidad contra lo que era de esperarse dadas las condiciones precarias en que se vivió curante esos años (vivienda, vestido, traumatismo psíquico), no así en materia de alimentación pues el gobierno con muy buen criterio crdenó todas las preferencias para las grávidas en materia de alimentación. El concepto de carencia fetal (Obes Polleri) (17) adquiere así singular importancia al establecer que la alimentación materna adecuada permite aún en presencia de otros facto- 
res adversos, obtener niños de peso óptimo para sobrevivir. Sin que pueda afirmarse que la carencia alimenticia provoque partos prematuros, sí puede decirse que con una alimentación ade. cuada, los demás factores de prematuridad tienen menos probabilidades de obrar y que aún en el caso de hacerlo, la mayor vitalidad del niño aún prematuro permite augurarle mejores perspectivas. En resumen, ante la imposibilidad de luchar exitosamente contra muchos de los factores médicos y económico-sociales, la alimentación de la madre en el último trimestre se muestra como un arma la más poderosa y accesible para por lo menos aumentar la proporción de "grandes prematuros" sobre los "pequeños prematuros" o inmaduros. Sabemos que la mortalidad del niño inmaduro es de $85 \%$ mientras que la del prematuro simpie es de solo $36 \%$. Es enormemente superior por otra parte la proporción de partos inmaduros en madres mal alimentadas en el último trimestre. Por la época del parto inmaduro el feto gana 200 gramos semanales aproximadamente. Vale la pena que el obste. tra se afine todo lo imaginable en la parte médica y se auxilie de la alimentación para obtener ese aumento. Con razón dice Obes Polleri: "Los 300 a 500 gramos que pueda ganar, significan para el iliño que va a nacer, la separación entre la vida y la muerte" (4).

Por otra parte, dentro de la alimentación, el factor más importante son las proteínas. Dentro de las 2.500 calorías que necesita la gestante, el suministro de proteínas no puede bajar de los 85 gramos representados en 1 litro de leche, 120 gramos de carne, 1 huevo y 30 gramos de queso. Dos observaciones cabe hacer en la necesidad del suministro de proteínas. Demostrada su importancia como factor de prematuridad y conocido el costo y eficacia solo parcial de los servicios para prematuros, cabe pensar si no resultaría más económico y benéfico para el Estado y las instituciones de beneficencia que atienden servicios obstétricos para la clase económicamente débil, establecer este suplemento nutritivo proteico para todas las madres que concurren a los servicios prenatales por lo menos en el último trimestre, lo cual por etra parte serviria de estímulo para su asistencia. La segunda observación se refiere al cuidado que' el obstetra debe poner cuando prescribe dietas a la embarazada por enfermedad general o por toxemia. La restricción proteica en la toxemia, por tanto tiempo practicada no se justifica más y agrava el problema de prematurez que conlleva esta complicación del embarazo. 
Enfrentados al hecho cumplido de que el niño ha de nacer prematuramente, la labor del médico consiste en hacer todos los esfuerzos por que los factores que producen mortalidad en el prematuro, actúen 10 menos intensamente posible. Vamos a analizar entonces el papel que el obstetra puede desempeñar en la atención del parto prematuro, en los cuidados inmediatos de este niño y los que habrá de prestársele en el curso de sus primeras 24 horas.

49. El parto del prematuro.-Una encuesta reaiizada entre 1.263 obstetras de los Estados Unidos (8) puso de manifiesto al. gunos dẹtalles sobre la forma más cientifica de conducir el parto prematuro. En materia de analgesia la mayoría fueron partidarios de no dar ninguna o si acaso una minima cantidad. La morfina y sus derivados o sucedáneos se consideran especialmente causales de respiración retardada, anoxia, etc. En cuanto a anestesia la mayoria abogan por ias anestesias regionales o locales (espinal, caudal, local). Especialmente para la cesárea que conlleva ya por las circunstancias que la indican, factores de anoxia, la anestesia raquídea es ia que puede brindar mayores seguridades para el prematuro. En relación con el manejo mismo del parto, la mayoria opina por el uso del forceps previa episiotomia cuando la cabeza llega al periné o bien forceps siempre que se prolonga el segundo período. Desde iuego, se recomienda la conservación de la bolsa de las aguas el mayor tiempo posible. Muchos son partidarios de no cortar el cordón inmediatamente sino esperar a que deje de pulsar y aún, exprimir sangre nacia el niño, como medida profiláctica contra la anemia del prematuro debida a sus bajas reservas en hierro y también como terapéutica transfusional para disminuir los efectos de la anoxia.

50 La atención inmediata del prematuro.-El despeje de las vias respiratorias de cuya importancia nadie duda ha sido some. tido a discusiones en relación con el método a seguir. El asunto parece fallado definitivamente en favor de los métodos más simples y suaves. Así la aspiración bucal por medio de un sencillo dispositivo provisto de cateter blando, ha venido a ser de nuevo el método de elección después de que los aspiradores eléctricos y las gruesas y rígidas sondas causaron muchos traumatismos en las frágiles mucosas del prematuro. En la mima sala de partos el niño debe ser colocado en una incubadora, con la cabeza más baja que los pies y en una atmósfera de $40 \%$ de oxígeno. 
En presencia de asfixia, de ninguna manera el éxito en el tratamiento de este accidente puede confiarse a la disponibilidad de tal o cual aparato mecánico de reanimación. Es el buen criterio y el "saber hacer" de un individuo capacitado lo que decide el resultado final. La mayoría de los procedimientos basados en la insuflación mecánica del pulmón por medio de presiones positivas, ocasionan más daños que beneficio (enfisema, neumotorax). La precipitación de un personal inexperto somete a procedimientos heróicos a niños simplemente adormecidos que hubieran respirado espontáneamente. La laringoscopia directa y el cateterismo traqueal son procedimientos delicados que solo deken ser practicados por un experto y solo a niños en estado de asfixia con flacidez (9) (14). Puede hacerse estimulación cutánea suave como método para estimular centros respiratorios inferiores y aún hasta flexiones de los muslos sobre el abdomen o presiones suaves sobre el torax. El aparato resucitador de Fletcher (10) (11) es recomendable por su sencillez e inocuidad. Algo que siempre debe hacerse en el prematuro es la aspiración endogástrica especialmente en los nacidos por cesárea. Lo que nunca debe hacerse es: presiones fuertes sobre el tórax, baños fríos y calientes, flexiones en "navaja" del cuerpo del niño, suspensiones prolongadas por los pies y movimiento de balanza. Los analépticos respiratorios deben usarse con cautela. Definitivamente parecen desechados por actuar en forma paradojal aumentando la inhibición, los de tipo central (Lobelina, Metrazol y Coramina). Si hay signos de Anoxia por estancamiento (cianosis que no cede en el oxígeno, trastorno circulatorio periférico) está indicada la inyección de adrenalina al 1 ) 0/00 de solución acuosa (11). Si se sospecha que la anoxia del niño sea debida a la administración a la madre de morfina y derivados del opio o sucedáneos, debe inyectarse dentro de la vena umbilical el clorhidrato de nalorphina (Nalline) a dosis de 0,1 mlg. en 2 c. c. de solución salina.

Otra de las medidas de la atención inmediata es la inyección de $5 \mathrm{mlg}$. de vitamina $\mathrm{K}$ para disminuír la tendencia hemorrágica.

Si la vitalidad del niño no parece buena es mejor no pesar1o, desvestirlo ni examinarlo innecesariamente.

60 Las primeras 24 horas del prematuro.-Colocado el niño como se ha dicho en una atmósfera de $40 \%$ de oxígeno y en posición de Trendelemburg (si no se sospecha hemorragia intracraneana) se practicará la aspiración de mucosidades por medio 
de una sonda suave y de preferencia con la boca. Si a pesar de naber practicado aspiración endogástrica en la sala de partos, hay excesivas secreciones luego, se puede hacer un lavado suave de estómago con 5 c. c. de solución salina. Si no presenta dificul. tades respiratorias o de hematosis el oxígeno puede irse disminuyendo gradualmente hasta llegar a la concentración ambiental de $20 \%$ al cabo de 12 o 24 horas para suspenderlo. Si hay falla en la actividad respiratoria está indicada la administración de $5 \%$ de gas carbónico en $95 \%$ de oxígeno por períodos de 2 minutos cada media hora. Puede repetirse la administración de cafeina o adrenalina cada 6 horas según se necesite.

La temperatura más usualmente usada en la incubadora es de 85 grados F. (32. $\left.3^{\circ} \mathrm{C}\right)$, con la cual algunos niños mantienen cierta hipotermia lo cual no debe preocupar si el niño por otros aspectos parece normal. Mucho más peligroso es el sobrecalentar1o, que agrava la anoxia y lo deshidrata. La temperatura de $90^{\circ} \mathrm{F}$ en la incubadora está indicada solo para los niños menores de 1.500 gramos que se vigilan más estrechamente. En general, la incubadora nunca debe tener una temperatura superior a $90^{\circ} \mathrm{F}$. La humedad no debe ser inferior a $65 \%$ y en casos patológicos (membrana hialina por ejemplo) debe elevarse hasta $910 \%$ por medio de' un nebulizador. El prematuro no debe colocarse en decúbito dorsal: estará siempre "sobre un lado y se le cambiará de posición cada 2 horas.

La administración de vitamina $K$ se proseguirá hasta que se establezca la alimentación oral (iniciación de su síntesis por la flora intestinal).

En general se establece que ningún niño prematuro debe recibir ningún alimento por boca antes de cumplir 24 horas de nacido. Los niños de 1.500 a 2.000 gramos deben esperar 36 horas y ios niños de menos de 1.000 gramos, hasta 60 horas. Estos tres grupos reciben solo un carbohidrato al 5\% durante 7,6 y 4 días respecitvamente. Los niños de más de 2.000 gramos pueden recibir leche desde el tercer día. Con buenas precauciones pueden hacerse algunas excepciones a la privación de alimento por más de 24 horas, en niños no asfícticos que muestran buena vitalidad. La presencia de cianosis, fatiga o cualquier otra dificultad en la alimentación imponen el uso de cateter para lo cual tanto el médico como el personal auxiliar deben tener buen entrenamiento. De ninguna manera puede pretenderse llenar desde un principio las necesidades de volumen, proteínas y calorías: tal intento so- 
lo conducirá a la gravísima complicación de la aspiración alimen. ticia; en esas condiciones es preferible habérselas con un niño subnutrido que no con bronconeumonía de aspiración.

yo Generalidades sobre atención de prematuros.- "Marfan" cuidaba exitosamente prematuros en una abadía del siglo XII" dice Pastor Oropeza en una publicación (12), para poner de presente que no cuenta tanto el edificio y la dotación como la preparación y suficiencia del personal que atiende prematuros. Puede decirse que es más importante la preparación técnica del personal auxiliar que la del mismo médico pues de nada sirve la labor de éste sin quien la ejecute adecuadamente. La proporción aceptada es la de una enfermera en cada turno de 8 horas para cada 5 niños prematuros, sin contar la enfermera jefe. Cuando el director de un servicio obstétrico sienta la tentación de construír un nuevo local o adquirir nuevos aparatos, debe detenerse a meditar si no resultará más benéfico nombrar más y mejor personal de enfermeras.

Pastor Oropeza en las recientes Jornadas Pediátricas de Cartagena expresó para sorpresa de los concurrentes, que en Vene. zuela habian llegado a la conclusión de que si insistían en atender a todos los prematuros de ese país en la forma como se viene haciendo en el Instituto Simón Rodríguez de Caracas, el respećable presupuesto de Venezuela se declararía impotente. Se esboza entonces la tendencia a atender el prematuro a domicilio lo más precozmente posible, llevando a su hogar, médico, enfer. mera y elementos y dando instrucción a la madre para constituirla en enfermera permanente. Por nuestra parte, en la Clínica David Restrepo de Bogotá hemos tomado la iniciativa bajando a 2.000 gramos el límite para egresar el prematuro si las condiciones del hogar lo permiten. Aún dentro de la misma clínica se trata de establecer la permanencia del niño con la madre. Si este "Rooming-In" como ha dado en llamarse, ha dado tan excelentes resultados en la disminución de la mortalidad neonatal en todos los países, no hay razón para pensar que el prematuro más grande por lo menos, no haya de beneficiarse, aliviando simultáneamente los servicios de prematuros que se reservarán para los más pequeños, que podrán ser así mucho mejor atendidos.

El obstetra como director de servicios de maternidad debe preocuparse por las cifras de mortalidad infantil en sus dependencias y el camino más seguro será ceñirse a las recomendaciores adoptadas internacionalmente en relación con la atención de recién nacidos y prematuros (13). 


\section{REFERENCIAS}

1. ILLPO, A.- "New classification and nomenclature for Newborn Infants including Prematures and Abortions". The Proceedings of the Eighth Northern Pediatric Congress 1946, in Helsinski, Finland; Acta Paediat. 1947.

2. A. M. A.--"Standard Nomenclature of Diseases and Operations".

3. BUNDESEN H. N.- "Natal Day Deaths. The Long-neglected field of Infant Mortality". J. A. M. A. 153: 466, oct. 3 de 1953.

4. OBES POLLERI.-“El Prematuro". P. 36. Ed. García Morales. Montevideo, Uruguay. 1952.

5. HAMMOND, J.-“Physiological factors affecting birth weight". Proceeding of the Nutrition Society; 2: 8. 1944.

6. WOOLF, B.-"Vital Statistics of Stillbirths and Neonatal Deaths". Brit. M. Bull.; 4: 170. 1946.

On the State of the Public Health During Six Years of War. Report of the Chicf Medical Officer of the Ministry of Health. 1939. London: His Majesty's Stationary Office; 1946.

Magee, H. E. Application of Nutrition to the Public Health. Some Lessons of War. Brit. Med. Journal; 475. 1945.

7. OBES POLLERI, J.- "Esquema del Prematuro". Actas y Trabajos del II Congreso Sudamericano de Pediatría. Pág. 459; El Ateneo, Buenos Aires. 1950.

Esquema del Prematuro. Arch. de Ped. del Uruguay; 20: 455. 1949.

8. LARSSON E.-“Saving the Premature Baby". Am. J. Obst. \& Gynec. 65: 158 165, January. 1953.

9. Role of the Pediatrician in Prevention of Needless Neonatal Deaths, J. A. M. A.: 153: 473, oct. 3 de 1953.

10. FLETCHER P. F. and ROGERS.-"Resusscitation of the Newborn". J. A. M. A. 145: 533, feb. 24 de 1951.

11. MILLAN FRANCISCO.-Tesis de grado. Facultad de Medicina de la Universidad Nacional. 1954.

12. FIGUEROA ERNESTO.-"Contribución al estudio de la organización y funcionamiento de un servicio de prematuros" (Instituto "Simón Rodríguez" Caracas, Venezuela).

13. American Accademy of Pediatrics: Standars and Recomendatios for the Hospital Care of Newborn Infant Full Term and Premature.

14. PLATA-RUEDA E.- "Organización del servicio para recién nacidos en el Hospital Central Militar de Bogotá," Colaboración para el Profesor Rafael Ramírez Merchán. 